\title{
MCP-1 Stimulates MMP-9 Expression via ERK 1/2 and p38 MAPK Signaling Pathways in Human Aortic Smooth Muscle Cells
}

\author{
Ci-Qiu Yanga Wen Lib,c Song-Qi Lia Jie Lia Yu-Wen Lid Shu-Xin Kong ${ }^{\mathrm{a}}$ \\ Rui-Ming Liüa,c Shen-Ming Wanga,c Wei-Ming Lva \\ aDivision of Vascular Surgery, 'baboratory of General Surgery, dDivision of Gastroenterology, The \\ First Affiliated Hospital, Sun Yat-sen University, Guangzhou, 'Guangdong Engineering Laboratory for \\ Diagnosis and Treatment of Vascular Disease, Guangzhou, China
}

\section{Key Words}

Abdominal aortic aneurysm $•$ Monocyte chemoattractant protein-1 $\bullet$ Matrix metalloproteinase- 9 - Human aortic vascular smooth cells - Extracellular signal regulated kinase - c-Jun amino terminal kinase $\bullet$ p38 mitogen activated protein kinases

\begin{abstract}
Objective: We investigated the molecular mechanism underlying the role of monocyte chemoattractant protein-1 (MCP-1) in the formation and development of human abdominal aortic aneurysm (AAA). Methods: We examined protein expression profiles using a protein array and found that MCP-1 was the most highly expressed protein in AAA tissues compared with normal aortas. To investigate the potential mechanism of MCP-1 involvement in the pathogenesis of AAA, we treated human aortic smooth muscle cells (HASMCs) with human recombinant MCP-1. Results: MCP-1 was the most highly expressed protein in AAA tissues compared with normal aorta; matrix metalloproteinase-9 (MMP-9) expression was also significantly increased. Treatment with MCP-1 significantly increased the expression and activation of MMP-9 and activated the three major mitogen activated protein kinases (MAPKs) extracellular signal regulated kinase (ERK), c-Jun amino terminal kinase (JNK1/2) and p38 MAPK. Furthermore, MCP-1-induced secretion of MMP-9 was inhibited by U0126 (inhibitor of the ERK 1/2 pathway) and SB203580 (inhibitor of the p38 MAPK pathway), but not SP600125 (inhibitor of the JNK1/2 pathway). Conclusion: These data demonstrate that MCP-1 stimulates secretion of MMP-9 directly through the ERK1/2 and p38 MAPK mediated pathways in HASMCs. Thus, inhibition of this molecular mechanism might be a potential therapeutic target in the non-surgical treatment of AAA.
\end{abstract}

C.-Q. Yang and W. Li contributed equally to this work. 


\section{Introduction}

Abdominal aortic aneurysm (AAA) is a common vascular disease and a leading cause of death that usually remains asymptomatic until rupture[1], which is an emergency with amortality rate of $80-90 \%$ [2]. Clinically, open or endovascular surgical repair is the only treatment for large $(>5.5 \mathrm{~cm})$ AAAs, and patients with aneurysms of diameter $<5.5 \mathrm{~cm}$ have no choice but to wait for the aneurysm to expand [3], making the non-operative management of AAA a complicated challenge.

AAA is a multifactorial degenerative disease [4] and decades of studies of the molecular basis of AAA have largely focused on changes in the integrity of the extracellular matrix (ECM) and chronic inflammatory infiltration ofthe aortic wall [5-8]. Matrix metalloproteinases (MMPs) have pivotal roles in these processes because their proteolytic activities assist in the degradation of the ECM and basement membrane, previous studies have reported that MMPs are expressed at low levels in healthy tissues but in the pathological state contribute to aortic wall expansion and subsequent AAA formation [4, 7, 9]. MMP-2 and MMP-9 are so far the most intensively investigated MMPs in human AAA, because they both possess significant proteolytic properties [10,11].

The other significant feature of AAA is that the aortic wall tissues are characterized by chronic inflammation with cellular infiltration. The inflammatory cellular response is to a large extent mediated by cytokines [12], which are secreted by these inflammatory cells and constitute a complex network that maintains the chronic inflammation in AAA. Previous studies have demonstrated the critical role of inflammatory cytokines in the pathogenesis of AAA through animal models. For example, it was demonstrated thataneurysm formation was attenuated in an elastase-induced rat AAA model by blocking the action of tumor necrosis factor- $\alpha$ (TNF- $\alpha$ ) [13]. Furthermore, inflammatory cells such as monocytes have an important role in AAA pathogenesis through the secretion and activation of MMPs [14].

To determine the molecular mechanism responsible for the formation and development of AAA, we used protein array analysis to generate 274 protein expression profiles for AAA tissues and normal aortas. This revealed that monocyte chemoattractant protein-1 (MCP1) was the most highly expressed protein in AAA tissues; expression of MMP-9 was also significantly greater in AAA than in controls. Previous studies have shown that MCP-1 is highly expressed in human AAA walls [15] and is increased in the aortic wall before the onset of chronic inflammatory responses and the development of AAA in mice [16], which indicates a pivotal role for MCP-1 in the formation and development of AAA.

Given the compelling data implicating MCP-1 in AAA and other inflammatory processes, it is important to establish whether human aortic smooth muscle cells (HSAMCs) respond to physiologic concentrations of MCP-1. In previous published results, Werle et al. [17] reported that MCP-1 induces secretion of MMP-2 in human endothelial cells and Tang et al. [18] showed that MCP-1 increases expression of MMP-9 in human chondrosarcoma cells. However, the molecular mechanism of the effect of MCP-1 on HASMCs remains unknown and the relationship between MCP-1 and MMPs in HASMCs has not been investigated. Here we show that MCP-1 may play an important role in the increased expression of MMP-9 in HASMCs; in addition, mitogen activated protein kinases (MAPKs) signaling pathways were found to be involved.

\section{Materials and Methods}

\section{Materials}

Human recombinant MCP-1 was purchased from Peprotech (Rocky Hill, NJ, USA). Anti-MCP-1, antiMMP-9, anti-p-extracellular signal regulated kinase (ERK)1/2, anti-ERK1/2, anti-p-c-Jun amino terminal kinase (JNK)1/2, anti-JNK1/2, anti-p-p38, anti-p38andU0126were purchasedfrom CellSignaling Technology (Beverly, MA, USA). Anti-smooth muscle $\alpha$-actin antibody and anti-MCP-1 for immunohistochemistry were purchased from Abcam (Cambridge, UK). SP600125 and SB203580 were purchased from Calbiochem (La 
Jolla, CA, USA). Cell culture media and fetal bovine serum (FBS) were purchased from Gibco BRL (Karlsruhe, Germany).

\section{Collection of specimens}

Full thickness AAA wall specimens were collected from 12 patients (seven men and five women) with an average age of 68.9 years who underwent elective surgical infrarenal repair between October 2011 and June 2012; adherent thrombus was removed manually at the time of surgery. Six non-aneurysmal abdominal aortic specimens obtained from cadaveric kidney donors were collected from age, sex and ethnicity matched controls. The samples were immediately snapped frozen in liquid nitrogen and processed for subsequent antibody array, western blot and quantitative polymerase chain reaction (qPCR) analysis; the remaining portions were fixed in $10 \%$ formalin for immunohistochemical analysis. This study was approved by the research ethics committee of the First Affiliated Hospital, Sun Yat-sen University (Permit Number: 2012274) and informed consent was given by all patients.

\section{Protein arrays}

The AAA and normal abdominal aortic specimens were analyzed using the Human Cytokine Array G4000 (AAH-CYT-G4000-8; RayBiotech, Inc., Norcross, GA, USA), which can detect 274 proteins. This protein array provides a relative expression level for each protein on the array when tested on a sample, measured in terms of the signal intensity and quantified by densitometry. The background of the array was used as the control to detect the presence of a protein in the sample.

\section{Cell culture}

HASMCs were isolated from the abdominal aorta of a healthy organ donor as described previously [19], with the consent of the donor and the approval of the ethics committee of the First Affiliated Hospital of Sun Yat-sen University. The HASMCs were maintained in Dulbecco's modified Eagle's medium (DMEM) supplemented with $10 \% \mathrm{FBS}$ and $1 \%$ penicillin/streptomycin at $37^{\circ} \mathrm{C}$ in a humidified atmosphere of $5 \% \mathrm{CO}_{2}$ and identified by staining with anti-smooth muscle $\alpha$-actin antibody. At confluence, HASMCs were serumstarved for $24 \mathrm{~h}$ and then treated with human recombinant MCP-1, U0126, SP600125 and SB203580 as indicated in each experiment. At the end of each treatment, conditioned media were collected for zymography and western blot analysis, and cells were collected for RNA extraction and western blot. HASMCs were used between passages three and six.

\section{Quantitative real time PCR}

Total RNA was extracted from HASMCs using RNAiso Plus (Takara, Dalian, China) according to the manufacturer's protocol. cDNA was synthesized using a PrimeScript RT reagent kit (Takara, Dalian, China) according to the manufacturer's instruction. Real time PCR was performed using an Applied Biosystems 7900HT Sequence Detection system with primers as follows: MMP-2 5'-CTCATCGCAGATGCCTGGAA-3' (forward), 5'-TTCAGGTAATAGGCACCCTTGAAGA-3' (reverse); MMP-9 5'-ACGCACGACGTCTTCCAGTA-3' (forward), 5'-CCACCTGGTTCAACTCACTCC-3' (reverse); and GAPDH 5'-GCACCGTCAAGGCTGAGAAC-3' (forward), 5'-TGGTGAAGACGCCAGTGGA-3' (reverse). Values obtained for target gene expression were normalized to GAPDH and quantified relative to their expression in the control samples.

\section{Western blot}

HASMCs were washed twice with ice-cold phosphate buffered saline (PBS) and whole cells were lysed using a protein extraction kit (Keygen, Nanjing, China) according to manufacturer's instructions. Equal amounts of total protein were separated by $10-15 \%$ sodium dodecyl sulfate polyacrylamide gel electrophoresis (SDS-PAGE) and subsequently transferred to polyvinylidene fluoride (PVDF) membranes (Roche, Basel, Switzerland). The blots were blocked with 5\% bovine serum albumin (BSA) for $1 \mathrm{~h}$ at room temperature and then probed with primary antibodies overnight at $4^{\circ} \mathrm{C}$. After three washes, the blots were incubated with horseradish peroxidase (HRP)-conjugated goat anti-mouse or -rabbit IgG for $1 \mathrm{~h}$ and subsequently visualized by enhanced chemiluminescence.

\section{Immunohistochemistry}

AAA tissues and normal aortas were fixed in 10\% formalin and embedded in paraffin. Briefly, after deparaffinization, rehydration, endogenous peroxidase activity blockage and antigen retrieval, sections 
Table 1. The ten most highly expressed proteins in abdominal aortic aneurysm (AAA) tissues versus normal aortas

\begin{tabular}{|c|c|c|c|c|c|c|}
\hline \multirow{2}{*}{$\begin{array}{l}\text { Spot } \\
\text { number }\end{array}$} & \multirow{2}{*}{$\begin{array}{l}\text { Official } \\
\text { symbol }\end{array}$} & \multirow[t]{2}{*}{ Protein name } & \multicolumn{2}{|c|}{ Relative expression signal intensity } & \multirow{2}{*}{$\begin{array}{l}\text { Fold } \\
\text { change }\end{array}$} & \multirow[t]{2}{*}{$P$} \\
\hline & & & AAA tissues & Normal aorta & & \\
\hline 1 & MCP-1 & Monocyte chemoattractant protein-1 & $50,388 \pm 17,682$ & $7820 \pm 7109$ & 6.44 & $<0.001$ \\
\hline 2 & RANTES & $\begin{array}{l}\text { Regulated on activation, normal } \mathrm{T} \text { cell } \\
\text { expressed and secreted }\end{array}$ & $35,483 \pm 24,061$ & $6227 \pm 6844$ & 5.70 & 0.002 \\
\hline 3 & FCGR2B & $\begin{array}{l}\text { Fc fragment of IgG, low affinity IIb, } \\
\text { receptor }\end{array}$ & $31,511 \pm 29,981$ & $3408 \pm 2007$ & 9.25 & 0.008 \\
\hline 4 & $M M P-8$ & $\begin{array}{l}\text { Matrix metallopeptidase- } 8 \text { (neutrophil } \\
\text { collagenase) }\end{array}$ & $22,842 \pm 14,517$ & $5165 \pm 3724$ & 4.42 & 0.001 \\
\hline 5 & $I L-6$ & Interleukin-6 (interferon beta 2) & $14,658 \pm 19,707$ & $0 \pm 0$ & & 0.026 \\
\hline 6 & Ferritin & Ferritin, mitochondrial & $14,620 \pm 6254$ & $2547 \pm 1284$ & 5.74 & $<0.001$ \\
\hline 7 & IGFBP-1 & $\begin{array}{l}\text { Insulin-like growth factor binding } \\
\text { protein } 1\end{array}$ & $13,724 \pm 12308$ & $491 \pm 766$ & 27.94 & 0.003 \\
\hline 8 & GDF-15 & Growth differentiation factor 15 & $12,685 \pm 7670$ & $4554 \pm 1039$ & 2.79 & 0.004 \\
\hline 9 & ICAM-1 & Intercellular adhesion molecule 1 & $10,141 \pm 6456$ & $1096 \pm 529$ & 9.25 & $<0.001$ \\
\hline 10 & CRP & C-reactive protein & $9779 \pm 3147$ & $5786 \pm 2230$ & 1.69 & 0.008 \\
\hline
\end{tabular}

were incubated overnight with anti-MCP-1 antibody and anti-MMP-9 antibody. The antigens were visualized using a SP Rabbit \& Mouse HRP kit (Kangwei, Beijing, China) according to the manufacturer's instructions. Finally, the sections were lightly counterstained with hematoxylin and analyzed under a microscope (BX51W1; Olympus, Tokyo, Japan).

\section{Zymography}

Conditioned media were concentrated fivefold by centrifugation through a Centricon Filter $(10,000$ molecular weight cut off; Millipore, Bedford, MA, USA) and mixed with SDS sample buffer without $\beta$-mercaptoethanol. The sample was loaded into $10 \%$ SDS-PAGE gels containing $0.1 \%$ of type I gelatin (Sigma-Aldrich, St. Louis, MO, USA) and electrophoresed at a constant voltage. Afterwards, the gel was washed with $2.5 \%$ Triton X-100 for 60 min to remove the SDS, incubated at $37^{\circ} \mathrm{C}$ for $48 \mathrm{~h}$ in a buffer $(50 \mathrm{mM}$ Tris-HCl, $\mathrm{pH} 8.8,5 \mathrm{mM} \mathrm{CaCl}_{2}, 1 \mu \mathrm{M} \mathrm{ZnCl}_{2}$ and $0.02 \%$ sodium azide $\mathrm{pH}$ 7.5) and stained with Coomassie Blue. The presence of gelatinolytic activity was identified as clear bands on a uniform blue background following destaining. The stained gels were visualized using a Gel Imaging System (G-BOX EF; Syngene, Cambridge, UK) and quantified by ImageJ2x Analysis software.

\section{Statistical analysis}

All analyses were performed using SPSS 17.0 software and GraphPad Prism 5.0. Data were presented as the mean \pm standard deviation and the significance of differences between the experimental groups and controls was assessed by Student's $t$ test. $P<0.05$ was considered to indicate a statistically significant difference.

\section{Results}

Microarray comparison of protein expression profile between AAA tissues and normal aortas

To further investigate the mechanisms of AAA formation, we performed a 274 protein array with AAA tissues from 12 AAA patients and normal aortas from 6 controls, 60 proteins were differentially expressed; $51 / 60$ proteins were increased and $9 / 60$ proteins were decreased in AAA tissues compared with normal aortas. The ten most highly expressed proteins (MCP-1, regulated on activation, normal T cell expressed and secreted (RANTES), Fc fragment of IgG, low affinity IIb, receptor(FCGR2B), MMP-8, interleukin-6 (IL-6), ferritin, insulin-like growth factor binding protein-1 (IGFBP-1), growth differentiation factor 15 (GDF-15), intercellular adhesion molecule 1 (ICAM-1) and C-reactive protein (CRP); Table 1) 


\section{A}
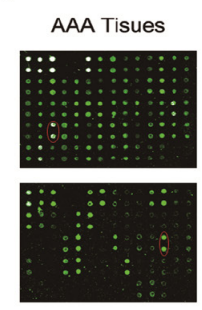

Normal Aortas

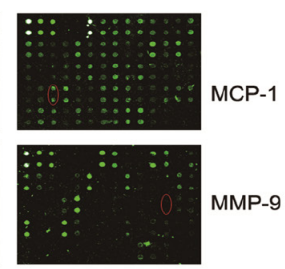

C

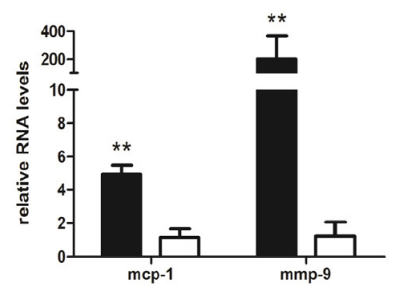

E

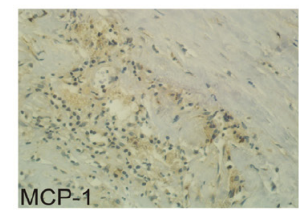

2
20

MMP-9

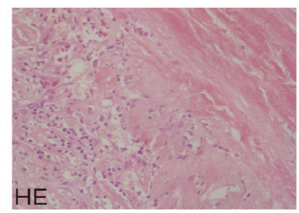

HE

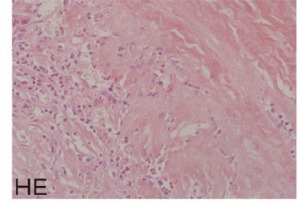

AAA tissues
B

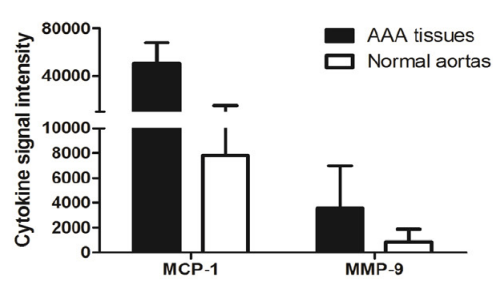

D
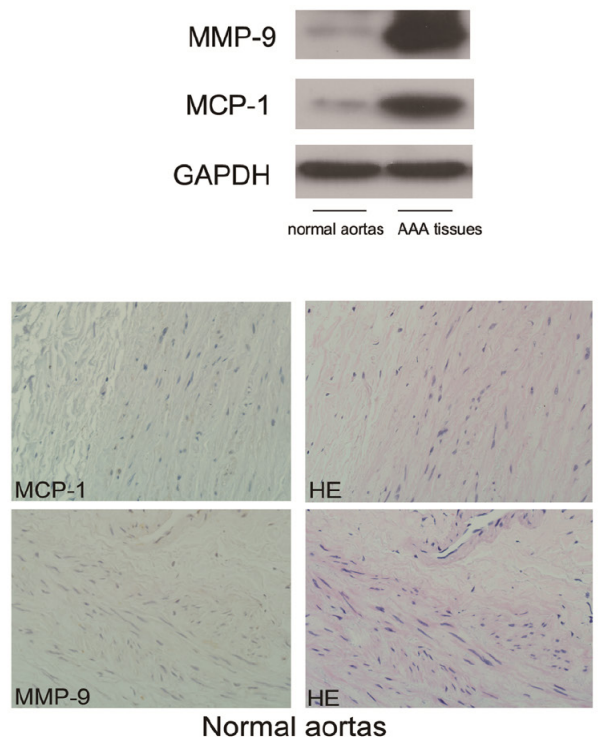

Fig. 1. Protein array and expression of monocyte chemoattractant protein-1 (MCP-1) and matrix metalloproteinase-9 (MMP-9) in abdominal aortic aneurysm (AAA) tissues and normal aortas. (A) Slides were scanned using a GenePix 4000B microarray scanner and image analysis was performed with GenePix Pro software (version 6.0). The pattern shown was a representative result. (B) Quantification of MCP-1 and MMP-9 in AAA tissues (black) and normal aorta (white). (C) Expression of MCP-1 and MMP-9 mRNA was detected by quantitative polymerase chain reaction analysis in AAA tissues and normal aorta. The intensity of bands was quantified and normalized to GAPDH. (D) MCP-1 and MMP-9 protein levels were measured by western blot analysis in AAA tissues and normal aorta. The intensity of bands was quantified and normalized to GAPDH. (E) Immunohistochemistry for MCP- 1 and MMP-9 and hematoxylin staining of AAA tissues and normal aortas (original magnification $\times 400$ ) ${ }^{*} P<0.05$, ${ }^{* *} P<0.01{ }^{* * *} P<0.001$ versus controls.

were all at significantly higher levels in AAA tissues; MCP-1 was the most highly expressed in AAA tissues compared with normal aortas $(50,388 \pm 17,682$ vs $7820 \pm 7109, P<0.001)$ and MMP-9 expression was also significantly greater in AAAs than in the controls (3568 \pm 3403 vs $842 \pm 1036, P=0.037$ ) (Fig. $1 \mathrm{~A}, \mathrm{~B}$ ).

\section{Elevated expression of MCP-1 and MMP-9 in AAA tissues}

The protein array data on MCP-1 and MMP-9 were further confirmed by qPCR and western blot. Expression of both MCP-1 and MMP-9 was significantly higher in AAA tissues than in normal aortas (Fig. 1C, D). On immunohistochemistry, AAA tissues stained positive for MCP-1 and MMP-9, whereas normal abdominal aorta showed little staining (Fig. 1E). 
A
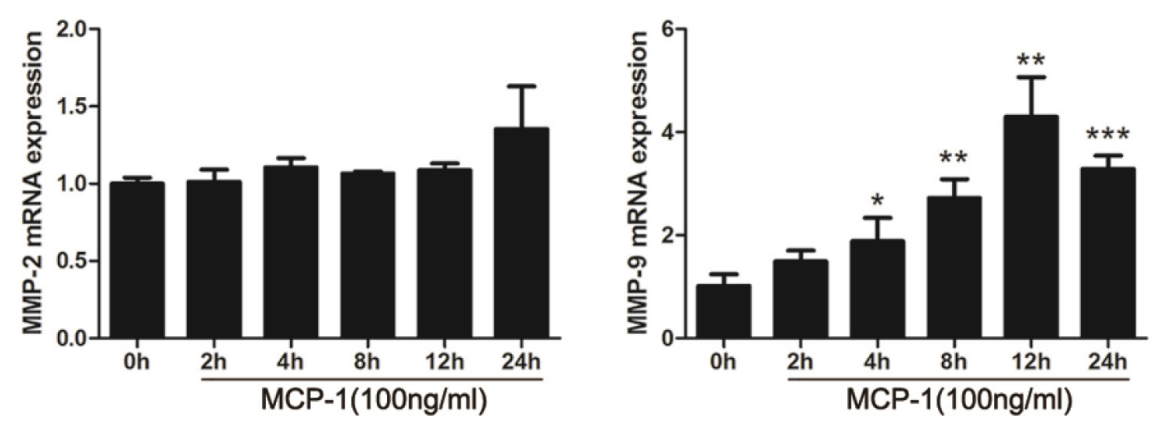

B

C
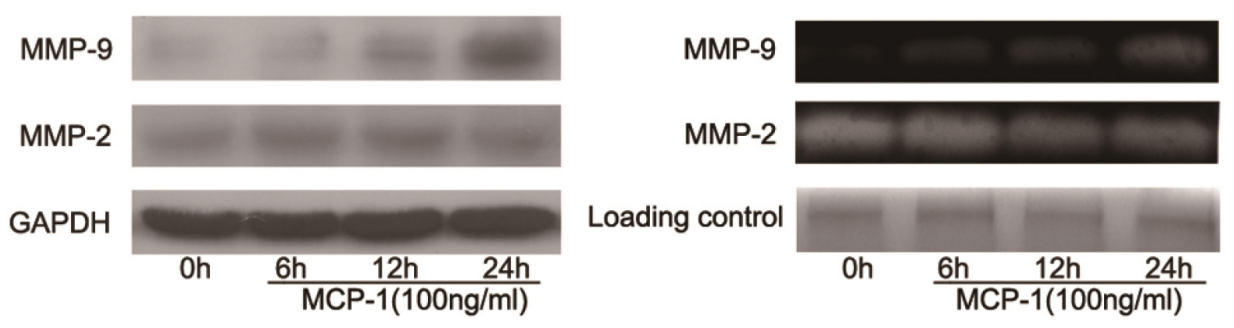

Fig. 2. Monocyte chemoattractant protein-1 (MCP-1) upregulated expression of matrix metalloproteinase-9 (MMP-9) but had no influence on MMP-2 in human aortic smooth muscle cells (HASMCs) incubated in $0.5 \%$ fetal bovine serum for $24 \mathrm{~h}$ and then treated with MCP-1. (A) HASMCs were incubated with MCP-1 for the indicated time intervals and MMP-2 and MMP-9 mRNA levels determined by quantitative polymerase chain reaction. (B) and (C) HASMCs were incubated with MCP-1 for the indicated time intervals. The culture medium and cell lysates were then collected. Levels of MMP-9 protein in cell lysates were measured by western blot analysis and the enzyme activities of MMP-2 and MMP-9 in the supernatant by zymography. ${ }^{*} P<0.05$, ${ }^{* *} P<0.01,{ }^{* * *} P<0.001$ versus controls.

\section{MCP-1-induced MMP-9 activity and expression in HASMCS}

Since expression of both MCP-1 and MMP-9 was upregulated in AAA tissues, we investigated whether there was any association between MCP-1 and MMP-9 induction in HASMCs. As shown in Fig. 2A, we treated HASMCs with human recombinant MCP-1 (100 $\mathrm{ng} / \mathrm{ml}$ ) from $0 \mathrm{~h}$ to $24 \mathrm{~h}$ and found that expression of MMP-9 was increased but expression of MMP-2 was unchanged on qPCR analysis. To further investigate the relationship between MCP-1 and MMP-9, the expression and activity of MMP-9 and MMP-2 were measured by western blot and zymography (Fig. 2B, C). We found that treatment of HASMCs with human recombinant MCP-1 (100 ng/ml) time dependently induced MMP-9 expression and activation but had no influence on MMP-2.

\section{MCP-1-induced activation of MAPKs ERK1/2, JNK1/2 and p38 MAPK in HASMCs}

It has been shown that MAPKs play a crucial role in the regulation of MMP-9 expression in human smooth muscle cells (SMCs) [20] and, as stated above, MCP-1 can induce the expression and activation of MMP-9; however, the relationship between MCP-1 and MAPKs in human SMCs had not been investigated previously. Thus, we first determined whether MCP-1 affects MAPK activity in HASMCs. As shown in Fig. 3A, MCP-1 (100 ng/ml) induced a rapid but transient increase of ERK 1/2, JNK 1/2 and p38 MAPK activity that started at 3 min and had almost returned to baseline levels within $2 \mathrm{~h}$. We then assessed the sensitivity of MAPK activation to the concentrationof MCP-1 (0.01-100 ng/ml). As shown in Fig. 3B, we found that a concentration of $100 \mathrm{ng} / \mathrm{ml}$ caused the greatest increase in ERK 1/2, JNK 1/2 
Fig. 3. Monocyte chemoattractant protein-1 (MCP-1) induced activation of the mitogen activated protein kinases (MAPKs) extracellular signal regulated kinase (ERK) 1/2, cJun amino terminal kinase (JNK) $1 / 2$ and p38 MAPK in human aortic smooth muscle cells (HASMCs) incubated in $0.5 \%$ fetal

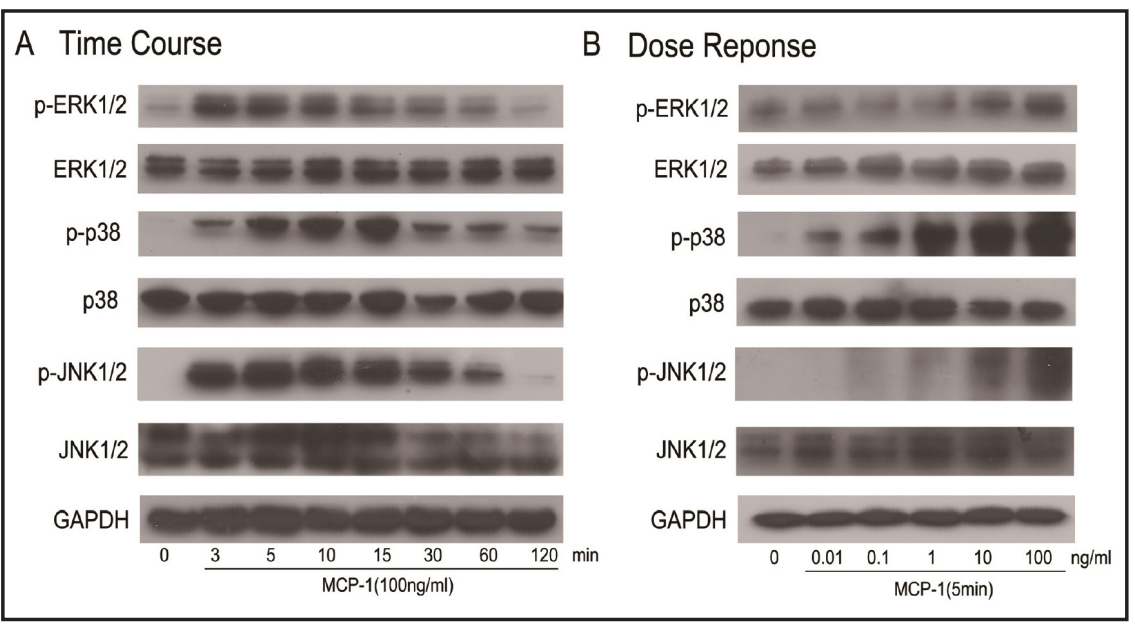
bovine serum for $24 \mathrm{~h}$ and then treated with MCP-1. (A) HASMCs treated with MCP-1 (100 ng/ml) for the indicated times (min). (B) HASMCs treated for $5 \mathrm{~min}$ with the indicated concentrations of MCP-1 (ng/ml).

Fig. 4. Monocyte chemoattractant protein-1 (MCP-1) upregulated matrix metalloproteinase-9 (MMP-9) via extracellular signal regulated kinase $1 / 2$ and p38 mitogen activated protein kinase. Human aortic smooth muscle cells were pretreated with U0126 $(10 \mu \mathrm{M})$, SP600125 $(25 \mu \mathrm{M})$ or SB203580 $(10 \mu \mathrm{M})$ for $1 \mathrm{~h}$ followed by further incubation for 12 or $24 \mathrm{~h}$ in the presence or absence of MCP-1. (A) Quantitative polymerase chain reaction analysis was performed. The values obtained for the target gene expression were normalized to GAPDH and quantified relative to the control samples. (B) and (C) MMP-9 and MMP-2 levels were determined by western blot analysis and zymography. The intensity of bands was quantified and normalized to GAPDH. ${ }^{* *} P<0.01$ versus control; ${ }^{\# \#} P<0.01$, \#\#\# $P<0.001$ versus MCP-1 treated group.

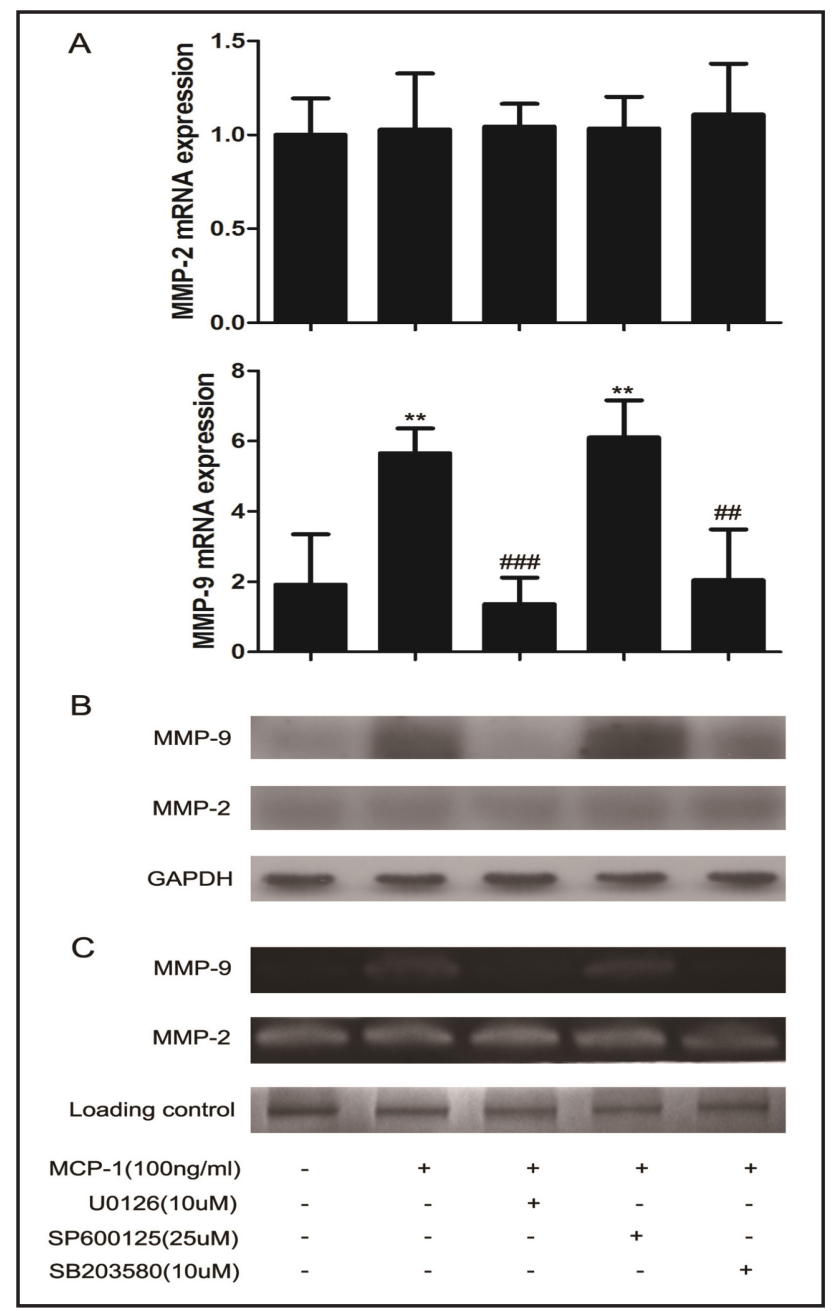

and p38 MAPK activation. In conclusion, MCP-1 caused time and concentration dependent increases in p-ERK1/2, p-JNK1/2 and p-p38 MAPK. 
ERK 1/2 and p38 MAPK but not JNK 1/2 mediated MCP-1-induced MMP-9 expression

To determine which cellular signaling pathway was involved in MCP-1-induced MMP-9 expression, HASMCs were stimulated with $100 \mathrm{ng} / \mathrm{ml} \mathrm{MCP-1}$ in the presence of U0126 (a MEK1/2 inhibitor) $(10 \mu \mathrm{M})$, SP600125 (a JNK1/2 inhibitor) $(25 \mu \mathrm{M})$ or SB203580 (a p38 MAPK inhibitor) $(10 \mu \mathrm{M})$. MMP-9 and MMP-2 levels were determined by qPCR, western blot and zymography. As shown in Fig. 4A, B, C, MMP-2 levels showed no change on treatment with MCP-1 and the three inhibitors. By contrast, MCP-1 treatment led to an obvious increase in MMP-9 levels that was completely abolished by U0126 and SB203580, but not by SP 600125 . These data demonstrate that ERK 1/2 and p38 MAPK are involved in MCP-1-induced MMP-9 expression in HASMCs.

\section{Discussion}

In the present study, we used protein array analysis to detect significant differences in the expression of 60 cytokines between AAA tissues and normal aortas, Proteins such as RANTES [21], MMP-8 [22], IL-6 [23] and IGFBP-1 [24] have been reported to play potentially important roles in the formation and development of AAA. Among these cytokines, we found MCP-1 to be the most highly upregulated protein in AAA tissues compared with normal aortas, and MMP-9 expression was also significantly higher in AAA. Previous studies have reported that MCP-1 exhibits biological activity in various cell types [17, 25]; however, nothing has been published previously regarding its effect on HASMCs and the intracellular signaling pathways involved. In the present study, treatment of HASMCs with MCP-1 apparently induced the activation of ERK1/2, JNK1/2 and p38 MAPK and increased the expression of MMP-9, which was attenuated by U0126 and SB203580 but not by SP 600125. These findings indicated that MCP-1 induced MMP-9 expression through the ERK1/2 and p38 MAPK mediated signaling pathways in HASMCs.

MCP-1 belongs to the CC chemokine family Invitro studies have demonstrated that numerous types of cell, including endothelial cells [26], SMCs [27], monocytes/ macrophages [28] and fibroblasts [29] arecapable of expressing MCP-1 in the presence of serum or specific stimuli. Previous studies have shown that MCP-1 is one of the most highly upregulated chemokines in human AAA walls [15] and it has been shown to be increased in AAA tissues, as described in an elastase-induced aneurysmal mouse [16]. The potential role for MCP-1 in AAA formation is supported by evidence that deletion of CCR2 prevents inflammatory responses and the development of AAA in mice [30]. Furthermore, MCP-1 is a multifunctional cytokine that promotes inflammation by numerous mechanisms and it is known that MCP-1 stimulates the activation of ERK 1/2, JNK 1/2 and p38 MAPK in human endothelial cells[17]. In the present study, we found MCP-1 to be the most highly expressed protein in AAA, and MCP-1 treatment promoted the activation of ERK 1/2, JNK 1/2 and p38 MAPK and upregulated MMP-9 expression in HASMCs.

Enzymatic degradation of the ECM is a crucial step in AAA formation and development [4]. MMP-2 and MMP-9 are involved in aneurysmal disease [31] and both are capable of degrading widespread matrix substrates in the ECM [32]. MMP-2 is constitutively expressed in several cell types including SMCs, but is not stimulated by cytokines or growth factors [33]; by contrast, MMP-9 is present at low levels under general conditions but its expression can be induced by treatment of cells with TNF- $\alpha$ or nerve growth factor [20, 34]. In accordance with previous studies, we found that MCP-1 induced MMP-9 expression but had no influence on MMP-2. MMP-9, also known as gelatinase B, plays a critical role in the degradation of ECM in AAA[14] and appears to be predominant in AAA in terms of mRNA expression. The ratio of MMP/tissue inhibitor of metalloproteinase is increased in AAA tissues compared with normal aortas [35] and upregulation of MMP-9 expression contributes to the degradation of widespread matrix substrates among these elastin [10,11]. Furthermore, animal studies have demonstrated that upregulation of MMP-9 contributes to the pathogenesis of aneurysms and plaque vulnerability by degrading ECM proteins [36, 37]. 
The MAPKs are a family of serine/threonine kinases that play an essential role in connecting cell-surface receptors to changes in transcriptional programs [38] associated with various cellular activities including cell proliferation, differentiation, survival, death and transformation [39-41]. The mammalian MAPK family comprises ERK1/2, JNK1/2 and p38 MAPK. Many previous studies have reported that the ERK1/2 and p38 MAPK pathways regulate MMP-9 expression. For example, Gum et al. [42] showed that overexpression of dominant-negative MEK 1 inhibits MMP-9 expression in phorbol myristate acetate (PMA)treated carcinoma cells. Using SB203580, Simon et al. [43] showed that p38 is involved in PMA-induced MMP-9 secretion. Cho et al. [20] showed that complete inhibition of either p38 MAPK or ERK $1 / 2$ alone or partial inhibition of both pathways totally downregulates MMP-9 expression. In accordance with these studies, using U0126 and SB203580, our data show that ERK 1/2 and p38 MAPK are involved in MCP-1-induced MMP-9 secretion. Based on these findings, we conclude that the expression of MMP- 9 induced by MCP-1 requires activation of ERK $1 / 2$ and p38 MAPK but not JNK 1/2 in HASMCs.

In conclusion, we provide direct evidence that expression of MCP-1 and MMP-9 is significantly upregulated in human AAA tissues compared with normal aortas. MCP-1 induces the activation of MAPK signaling pathways and upregulates the expression of MMP-9 through the ERK1/2 and p38 MAPK signaling pathways, which may cause proteolytic degradation of the aortic wall and increase the susceptibility of the aneurysm to expansion and rupture. Further investigations into the functional significance of the individual cytokines altered in the aneurysmal wall will be crucial to increasing our understanding of AAA and fostering clinical improvements.

\section{Acknowledgments}

This study was supported by grants from the National Natural Science Foundation of China (Nos. 81172337, 81370368, 30973395) and the Key Clinical Program of the Ministry of Health.

\section{Disclosure Statement}

The authors declare that they have no conflict of interest.

\section{References}

1 Krupski WC, Rutherford RB: Update on open repair of abdominal aortic aneurysms: the challenges for endovascular repair. J Am Coll Surg 2004;199:946-960.

-2 Cornuz J, Sidoti Pinto C, Tevaearai H, Egger M: Risk factors for asymptomatic abdominal aortic aneurysm: systematic review and meta-analysis of population-based screening studies. Eur J Public Health 2004;14:343-349.

- 3 Nanda S, Sharma SG, Longo S: Molecular targets and abdominal aortic aneurysms. Recent Pat Cardiovasc Drug Discov 2009;4:150-159.

4 Saratzis A, Kitas GD, Saratzis N, Melas N: Can statins suppress the development of abdominal aortic aneurysms? A review of the current evidence. Angiology 2010;61:137-144.

5 Eagleton MJ: Inflammation in abdominal aortic aneurysms: cellular infiltrate and cytokine profiles. Vascular 2012;20:278-283.

6 Abdul-Hussien H, Soekhoe RG, Weber E, von der Thusen JH, Kleemann R, Mulder A, van Bockel JH, Hanemaaijer R, Lindeman JH: Collagen degradation in the abdominal aneurysm: a conspiracy of matrix metalloproteinase and cysteine collagenases. Am J Pathol 2007;170:809-817.

7 Saratzis A, Abbas AA, Kiskinis D, Melas N, Saratzis N, Kitas GD: Abdominal aortic aneurysm: a review of the genetic basis. Angiology 2011;62:18-32. 
Yang et al.: Yang MCP-1 Stimulates MMP-9 Expression in HASMCs

8 Anidjar S, Dobrin PB, Eichorst M, Graham GP, Chejfec G: Correlation of inflammatory infiltrate with the enlargement of experimental aortic aneurysms. J Vasc Surg 1992;16:139-147.

-9 Raffetto JD, Khalil RA: Matrix metalloproteinases and their inhibitors in vascular remodeling and vascular disease. Biochem Pharmacol 2008;75:346-359.

10 Papalambros E, Sigala F, Georgopoulos S, Menekakos C, Giatromanolaki A, Bastounis E, Sivridis E: Immunohistochemical expression of metalloproteinases MMP-2 and MMP-9 in abdominal aortic aneurysms: correlation with symptoms and aortic diameter. Int J Mol Med 2003;12:965-968.

$\checkmark 11$ Petersen E, Gineitis A, Wagberg F, Angquist KA: Activity of matrix metalloproteinase-2 and -9 in abdominal aortic aneurysms. Relation to size and rupture. Eur J Vasc Endovasc Surg 2000;20:457-461.

12 Jones KG, Brull DJ, Brown LC, Sian M, Greenhalgh RM, Humphries SE, Powell JT: Interleukin-6 (IL-6) and the prognosis of abdominal aortic aneurysms. Circulation 2001;103:2260-2265.

13 Hingorani A, Ascher E, Scheinman M, Yorkovich W, DePippo P, Ladoulis CT, Salles-Cunha S: The effect of tumor necrosis factor binding protein and interleukin-1 receptor antagonist on the development of abdominal aortic aneurysms in a rat model. J Vasc Surg 1998;28:522-526.

14 Thompson RW, Holmes DR, Mertens RA, Liao S, Botney MD, Mecham RP, Welgus HG, Parks WC: Production and localization of 92-kilodalton gelatinase in abdominal aortic aneurysms. An elastolytic metalloproteinase expressed by aneurysm-infiltrating macrophages. J Clin Invest 1995;96:318-326.

15 Kudo J, Yoshimura K, Hamano K: Simvastatin reduces secretion of monocyte chemoattractant proteins and matrix metalloproteinase-9 in human abdominal aortic aneurysms. The bulletin of the Yamaguchi Medical School 2007;54:47-56.

-16 Colonnello JS, Hance KA, Shames ML, Wyble CW, Ziporin SJ, Leidenfrost JE, Ennis TL, Upchurch GR Jr, Thompson RW: Transient exposure to elastase induces mouse aortic wall smooth muscle cell production of MCP-1 and RANTES during development of experimental aortic aneurysm. J Vasc Surg 2003;38:138-146.

-17 Werle M, Schmal U, Hanna K, Kreuzer J: MCP-1 induces activation of MAP-kinases ERK, JNK and p38 MAPK in human endothelial cells. Cardiovasc Res 2002;56:284-292.

18 Tang CH, Tsai CC: CCL2 increases MMP-9 expression and cell motility in human chondrosarcoma cells via the Ras/Raf/MEK/ERK/NF-kappaB signaling pathway. Biochem Pharmacol 2012;83:335-344.

19 Wang M, Li W, Chang GQ Ye CS, Ou JS, Li XX, Liu Y, Cheang TY, Huang XL, Wang SM: MicroRNA-21 regulates vascular smooth muscle cell function via targeting tropomyosin 1 in arteriosclerosis obliterans of lower extremities. Arterioscler Thromb Vasc Biol 2011;31:2044-2053.

20 Cho A, Graves J, Reidy MA: Mitogen-activated protein kinases mediate matrix metalloproteinase-9 expression in vascular smooth muscle cells. Arterioscler Thromb Vasc Biol 2000;20:2527-2532.

21 Houard X, Touat Z, Ollivier V, Louedec L, Philippe M, Sebbag U, Meilhac O, Rossignol P, Michel JB: Mediators of neutrophil recruitment in human abdominal aortic aneurysms. Cardiovasc Res 2009;82:532-541.

22 Wilson WR, Anderton M, Schwalbe EC, Jones JL, Furness PN, Bell PR, Thompson MM: Matrix metalloproteinase- 8 and -9 are increased at the site of abdominal aortic aneurysm rupture. Circulation 2006;113:438-445.

23 Rohde LE, Arroyo LH, Rifai N, Creager MA, Libby P, Ridker PM, Lee RT: Plasma concentrations of interleukin- 6 and abdominal aortic diameter among subjects without aortic dilatation. Arterioscler Thromb Vasc Biol 1999;19:1695-1699.

-24 Ramos-Mozo P, Rodriguez C, Pastor-Vargas C, Blanco-Colio LM, Martinez-Gonzalez J, Meilhac O, Michel JB, Vega de Ceniga M, Egido J, Martin-Ventura JL: Plasma profiling by a protein array approach identifies IGFBP-1 as a novel biomarker of abdominal aortic aneurysm. Atherosclerosis 2012;221:544-550.

25 Yamamoto T, Eckes B, Mauch C, Hartmann K, Krieg T: Monocyte chemoattractant protein-1 enhances gene expression and synthesis of matrix metalloproteinase-1 in human fibroblasts by an autocrine IL-1 alpha loop. J Immunol 2000;164:6174-6179.

26 Rollins BJ, Yoshimura T, Leonard EJ, Pober JS: Cytokine-activated human endothelial cells synthesize and secrete a monocyte chemoattractant, MCP-1/JE. Am J Pathol 1990;136:1229-1233.

27 Valente AJ, Graves DT, Vialle-Valentin CE, Delgado R, Schwartz CJ: Purification of a monocyte chemotactic factor secreted by nonhuman primate vascular cells in culture. Biochemistry 1988;27:4162-4168.

28 Yoshimura T, Robinson EA, Tanaka S, Appella E, Leonard EJ: Purification and amino acid analysis of two human monocyte chemoattractants produced by phytohemagglutinin-stimulated human blood mononuclear leukocytes. J Immunol 1989;142:1956-1962. 
Yang et al.: Yang MCP-1 Stimulates MMP-9 Expression in HASMCs

29 Strieter RM, Wiggins R, Phan SH, Wharram BL, Showell HJ, Remick DG, Chensue SW, Kunkel SL: Monocyte chemotactic protein gene expression by cytokine-treated human fibroblasts and endothelial cells. Biochem Biophys Res Commun 1989;162:694-700.

30 Ishibashi M, Egashira K, Zhao Q, Hiasa K, Ohtani K, Ihara Y, Charo IF, Kura S, Tsuzuki T, Takeshita A, Sunagawa K: Bone marrow-derived monocyte chemoattractant protein-1 receptor CCR2 is critical in angiotensin II-induced acceleration of atherosclerosis and aneurysm formation in hypercholesterolemic mice. Arterioscler Thromb Vasc Biol 2004;24:e174-178.

-31 Newman KM, Malon AM, Shin RD, Scholes JV, Ramey WG, Tilson MD: Matrix metalloproteinases in abdominal aortic aneurysm: characterization, purification, and their possible sources. Connect Tissue Res 1994;30:265-276.

-32 Thompson RW, Parks WC: Role of matrix metalloproteinases in abdominal aortic aneurysms. Ann N Y Acad Sci 1996;800:157-174.

33 Galis ZS, Muszynski M, Sukhova GK, Simon-Morrissey E, Unemori EN, Lark MW, Amento E, Libby P: Cytokine-stimulated human vascular smooth muscle cells synthesize a complement of enzymes required for extracellular matrix digestion. Circ Res 1994;75:181-189.

-34 Khan KM, Falcone DJ, Kraemer R: Nerve growth factor activation of Erk-1 and Erk-2 induces matrix metalloproteinase-9 expression in vascular smooth muscle cells. J Biol Chem 2002;277:2353-2359.

35 Tamarina NA, McMillan WD, Shively VP, Pearce WH: Expression of matrix metalloproteinases and their inhibitors in aneurysms and normal aorta. Surgery 1997;122:264-271; discussion 271-262.

-36 Pyo R, Lee JK, Shipley JM, Curci JA, Mao D, Ziporin SJ, Ennis TL, Shapiro SD, Senior RM, Thompson RW: Targeted gene disruption of matrix metalloproteinase-9 (gelatinase B) suppresses development of experimental abdominal aortic aneurysms. J Clin Invest 2000;105:1641-1649.

-37 Petrinec D, Liao S, Holmes DR, Reilly JM, Parks WC, Thompson RW: Doxycycline inhibition of aneurysmal degeneration in an elastase-induced rat model of abdominal aortic aneurysm: preservation of aortic elastin associated with suppressed production of 92 kD gelatinase. J Vasc Surg 1996;23:336-346.

38 Chang L, Karin M: Mammalian MAP kinase signalling cascades. Nature 2001;410:37-40.

-39 McCubrey JA, Lahair MM, Franklin RA: Reactive oxygen species-induced activation of the MAP kinase signaling pathways. Antioxid Redox Signal 2006;8:1775-1789.

40 Torii S, Yamamoto T, Tsuchiya Y, Nishida E: ERK MAP kinase in G cell cycle progression and cancer. Cancer Sci 2006;97:697-702.

41 Dhillon AS, Hagan S, Rath O, Kolch W: MAP kinase signalling pathways in cancer. Oncogene 2007;26:32793290.

42 Gum R, Wang H, Lengyel E, Juarez J, Boyd D: Regulation of 92 kDa type IV collagenase expression by the jun aminoterminal kinase- and the extracellular signal-regulated kinase-dependent signaling cascades. Oncogene 1997;14:1481-1493.

43 Simon C, Goepfert H, Boyd D: Inhibition of the p38 mitogen-activated protein kinase by SB 203580 blocks PMA-induced Mr 92,000 type IV collagenase secretion and in vitro invasion. Cancer Res 1998;58:11351139. 